\title{
Charge Generation and Collection in p-n Junctions Excited with Pulsed Infrared Lasers
}

\author{
A. H. Johnston \\ Jet Propulsion Laboratory \\ California Institute of Technology \\ Pasadena, California 91109
}

\begin{abstract}
Optical absorption processes are examined for applications of infrared lasers to simulate single-particle effects in silicon and GaAs, which require that the laser is focused to a small area on the device surface. The resulting charge generation is compared with charge generation from heavy ions. Charge funneling is reduced in silicon structures because of the lower charge density unless the LET is above a threshold value. In both materials, the effective LET of a laser is inherently non-linear because of nonlinear absorption at high intensities. These factors limit quantitative comparisons between lasers and heavy ions, and are increasingly important as devices are scaled to smaller dimensions.

INTRODUCTION
\end{abstract}

Pulsed infrared lasers have been used to simulate a variety of pulsed ionization effects in semiconductors, [1-5] including single-particle effects from heavy ions. Lasers are extremely useful as diagnostic tools, providing the ability to precisely locate the laser beam position on a test device, which allows determination of the responses of specific internal regions of a complex circuit to transient pulses. A laser pulse can also be synchronized with external equipment, providing temporal information that cannot be obtained from heavy-ion testing. Although lasers provide unique advantages as diagnostic tools, there are fundamental differences in the ionization process and the resulting charge density compared to heavy ions, and questions remain about how to calibrate them and how to compare laser responses with other test approaches.

This paper examines several aspects of laser simulation that have not been addressed in previous work, and relates them to device processing and scaling. There are mechanisms that cause the effective absorption to be nonlinear with depth and light intensity, particularly for single-particle effects where a high-intensity laser is focused on a highly localized region. The resulting carrier distribution, which is also affected by optical resolution limitations, is substantially different from the carrier distribution that occurs after the interaction of a heavy charged particle. This reduces charge funneling, and also affects phenomena that depend on diffused charge, such as latchup and multiple-bit upset. These effects, along with the change in absorption coefficient associated with bandgap narrowing, provide additional limitations in the ability to make absolute comparisons between laser studies and tests with heavy-ion sources. Although they do not diminish the effectiveness of the laser as a diagnostic tool, they must be taken into account when comparing laser test results with heavy-ion tests.

The first section of the paper discusses fundamental optical absorption in semiconductors, the assumptions involved in establishing equivalence between lasers and heavy ions, and the effect of bandgap narrowing. Section two compares carrier densities generated by lasers and heavy ions, noting that charge funneling is reduced or eliminated at higher doping levels and low LET values when a laser is used, in contradiction to earlier work. This is followed by a discussion of nonlinear absorption processes in section three. Section four discusses applications of lasers to specific device types and mechanisms, showing how nonlinear absorption and differences in funneling affect laser results, followed by summary and conclusions.

\section{BAND-TO-BAND ABSORPTION}

Wavelength Selection. Most experimental work with lasers has assumed that the light is absorbed by a fundamental band-to-band absorption process, producing ionization by exciting carriers from the valence to the conduction band. Each absorbed photon is assumed to produce a single electronhole pair. The light is then absorbed exponentially with depth, as described by Beer's law: $\mathrm{I}=\mathrm{I}_{0} \mathrm{e}^{-\alpha x}$. The absorption coefficient, $\alpha$, depends strongly on wavelength, as shown in Figure 1 for lightly doped silicon and GaAs.[6-9] The wavelength of the laser must be selected to provide sufficiently uniform penetration to simulate the effect of heavy ions over the charge collection distance associated with the device technology. The charge collection depth is strongly affected by device construction, as well as the particular phenomenon that is being studied. For single-event upset (SEU) in shallow silicon structures, the collection depth may be only a few microns, whereas for latchup or multiple-bit upset, charge collection depths may extend to nearly $100 \mu \mathrm{m}$ because of the importance of charge diffusion from extended regions in the substrate. Most GaAs structures have shallow junctions, but charge trapping effects in semi-insulating GaAs substrates and the presence of parasitic bipolar structures in MESFETs have been shown to be important in determining SEU effects in GaAs.[10] Much deeper penetration depths are required to simulate these mechanisms. 


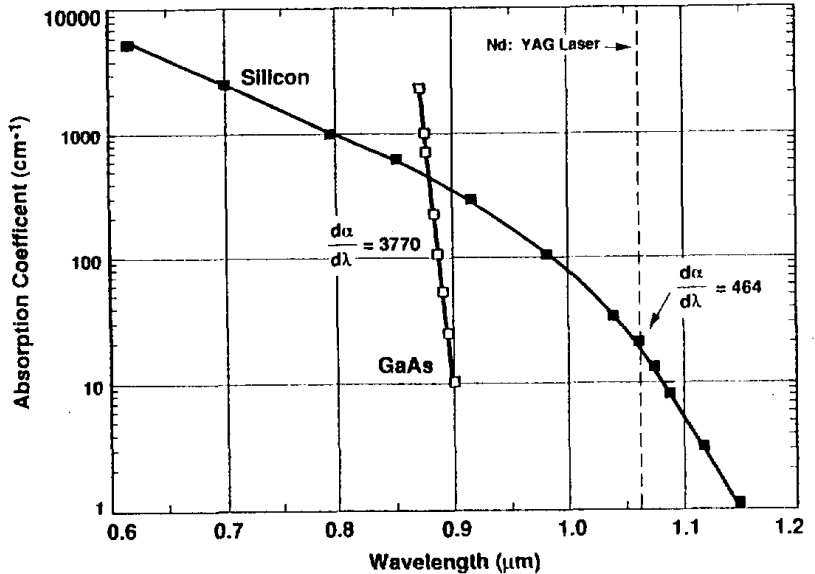

Figure 1. Absorption Coefficient in Lightly Doped Silicon and Gallium Arsenide.

Figure 1 includes calculations of the slope of the absorption coefficient, $\mathrm{d} \alpha / \mathrm{d} \lambda$, in $\mathrm{cm} / \mu \mathrm{m}$, near the wavelengths that are typically used for the two materials. Because GaAs is a direct bandgap material, its absorption coefficient has a much steeper dependence on wavelength, approximately two orders of magnitude greater than that of silicon. This makes it more difficult to select a suitable wavelength. Although tunable dye lasers are usually used for GaAs, other factors such as bandgap narrowing make it difficult to get uniform penetration in different regions of GaAs structures because the absorption coefficient depends on doping concentration. This will be discussed in a later section.

It is much easier to select a laser wavelength for silicon because of the shallower slope of the absorption coefficient. The Nd:YAG laser $(\lambda=1.06 \mu \mathrm{m})$ is nearly ideal, with a penetration depth of about $700 \mu \mathrm{m}$. Most work with silicon has been done using this type of laser. Nd:YAG lasers have very high power levels compared to tunable dye lasers, making it easy to get high effective LET values with low $\alpha$. It is also possible to use other wavelengths for silicon, including frequency-doubled Nd:YAG $(0.532 \mu \mathrm{m})$, or intermediate wavelengths from tunable lasers. However, lasers with wavelengths below $0.8 \mu \mathrm{m}$ have penetration depths in silicon that are much less than that required for heavy-ion testing, and should generally be avoided because the ionization track is confined to the top surface. Although it is possible to produce upsets with short-wavelength lasers, the charge distribution is so different from that of heavy. ions that it is virtually impossible to obtain quantitative comparisons with heavy ion tests. Thus, their are two basic requirements for laser simulation of single-particle effects: first, the wavelength must be selected to provide a penetration depth of $10-100 \mu \mathrm{m}$, depending on the phenomenon that is being simulated; and second, the pulse length of the laser should be short compared to the charge collection time in the depletion and funneling region. Typical pulse lengths are 15-25 ps.

Equivalent Laser Energy. The usual way to apply lasers for single-particle applications is to equate the number of photons absorbed by the laser in a thin region to the number of carriers generated by the track of a heavy charged particle. For laser wavelengths with energies near the bandgap energy, this assumes that one electron-hole pair is created by each absorbed photon. Disregarding surface reflection, and assuming that the penetration depth is uniform over the depth of interest, this can be expressed as

$$
E_{\Lambda}=\frac{10^{-15} \text { h cLET* }^{*}}{\alpha \lambda \mathrm{q}}
$$

where $E_{\Lambda}$ is the incident laser pulse energy $(J), h$ is Planck's constant, $\mathrm{c}$ is the velocity of light, $\alpha$ is the absorption coefficient $\left(\mathrm{cm}^{-1}\right), \lambda$ is the laser wavelength $(\mathrm{cm})$, LET $^{*}$ is linear-energy transfer ${ }^{\dagger}$ in $\mathrm{pC} / \mu \mathrm{m}$, and $\mathrm{q}$ is electronic charge. The factor of $10^{-15}$ converts to mks units. For LET ${ }^{*}=0.1$ $\left(\mathrm{LET}=9.8 \mathrm{MeV}-\mathrm{cm}^{2} / \mathrm{mg}\right)$, the incident laser energy for a focused Nd:YAG laser in silicon is $4.6 \times 10^{-11} \mathrm{~J} /$ pulse.

The power density of a focused laser is quite high. For an energy equivalent to LET $* 0.1$, a mode-locked laser with a pulse length of $15 \mathrm{ps}$, focused to a spot size of $2 \mu \mathrm{m}^{2}$ produces an average power density during the laser pulse of 0.15 $\mathrm{GW} / \mathrm{cm}^{2}$, an extremely high value. As discussed later, this power level is sufficiently high so that nonlinear optical processes become significant, changing the absorption coefficient.

Although it is often not explicitly stated, band-to-band absorption with a constant absorption coefficient has been assumed in nearly all previous work on laser simulation. In many cases, particularly for older device technologies, this fundamental absorption accurately describes laser absorption. However, the assumption of linear absorption is not necessarily valid for newer silicon-based technologies and most GaAs technologies. The mechanisms and conditions under which absorption becomes nonlinear are discussed further in later sections of the paper.

Temperature Dependence. The absorption coefficient depends on temperature. For silicon, $\alpha$ approximately doubles - . . . . . . . . .

†Using LET* $(\mathrm{pC} / \mu \mathrm{m})$ simplifies the application of Equation 1 to materials other than silicon. For silicon, LET* can be converted to LET in $\mathrm{MeV}-\mathrm{cm}^{2} / \mathrm{mg}$ by multiplying by 98 . For GaAs, the conversion factor is 57 . 
at $125 \mathrm{C}$ compared to its value at room temperature. This affects the equivalent LET of laser pulses when devices are tested at elevated temperature, and must be accounted for when devices are tested at temperatures other than room temperature. This is particularly important for phenomena such as latchup, which have increased susceptibility at elevated temperature.[11] Although the bandgap temperature dependence can be used for approximate corrections to room temperature absorption, there are a number of temperaturedependent phenomena that can affect results in addition to the absorption coefficient, such as resistivity and minority carrier lifetime. This limits the effectiveness of indirect calibration by correlation with heavy-ion test results, and makes it difficult to study temperature-dependent effects with lasers.

Bandgap Narrowing. At high doping levels, the band structure is modified because of the large number of localized impurity states near the band edge.[12] This effect, known as band tailing, reduces the energy gap (and hence changes the absorption coefficient) as the impurity level increases. This is extremely important because it causes the absorption coefficient, and hence the effective LET of an incident laser pulse to vary in different regions of a semiconductor device structure. It has a pronounced effect on laser calibration and correlation, and has been overlooked by many previous workers. Bandgap narrowing is particularly important in GaAs because of the very steep slope of the absorption coefficient. The effect of bandgap narrowing on the absorption coefficient of $p$ - and n-type gallium arsenide is shown in Figures 2 and 3.[13] Note that the absorption coefficient near the band edge changes by more than one order of magnitude at high doping concentrations. For GaAs, the absorption coefficient of p-material increases with doping level, whereas $\alpha$ decreases with doping level for $n$-material. In p-GaAs, concentrations as low as $2 \times 10^{17} \mathrm{~cm}^{-3}$ cause

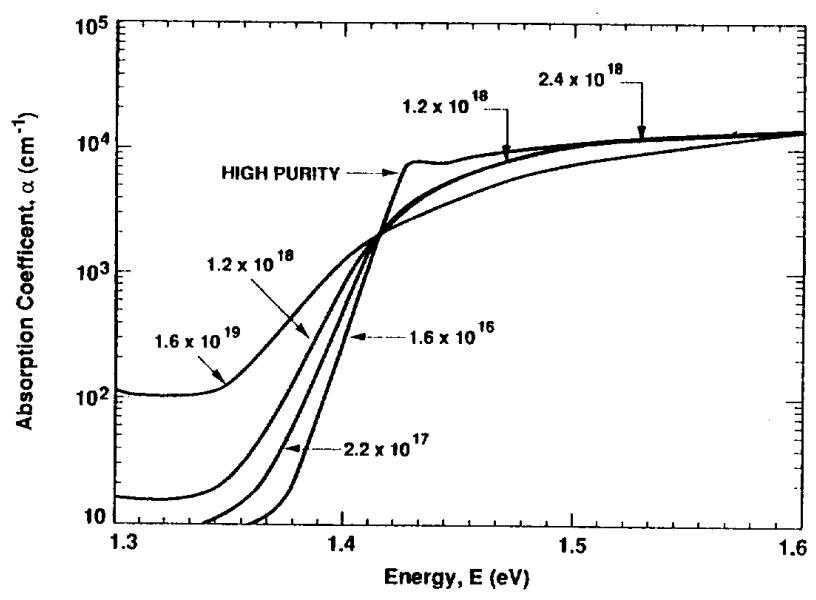

Figure 2. Bandgap Narrowing in p-Type GaAs (after Casey, Sell, and Wert, Reference 13). significant changes in the absorption coefficient. In n-GaAs the doping concentration must be about an order of magnitude higher before $\alpha$ is affected significantly. Many GaAs structures use doping levels of this magnitude, causing the absorption coefficient to be much different than that of lightly doped regions, such as semi-insulating substrates.

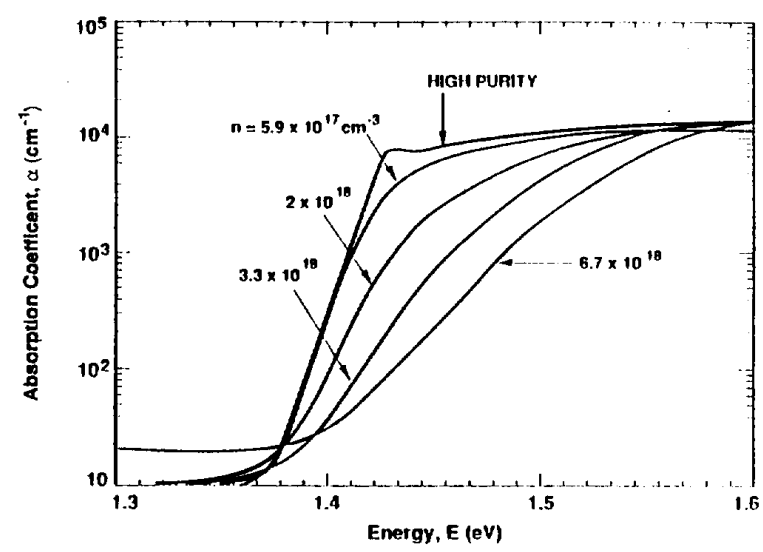

Figure 3. Bandgap Narrowing in n-Type GaAs (after Casey, Sell, and Wert, Reference 13).

Bandgap narrowing is also important in silicon. Figure 4 compares the absorption coefficient of silicon at several different doping levels for p-doped material.[14] The absorption coefficient at $1.06 \mu \mathrm{m}$ doubles when the doping concentration is about $3 \times 10^{18} \mathrm{~cm}^{-3}$, and continues to increase as the doping level increases. In older technologies, doping levels above $10^{18} \mathrm{~cm}^{-3}$ were used only in shallow regions near the top surface (such as source/drain and well contact diffusions) where the increases in absorption coefficient had little effect on SEU-related charge. However, newer device technologies may use highly doped regions in deeper regions, increasing the importance of bandgap narrowing for scaled devices.[15] For example, highly doped p-substrates are used on many epitaxial CMOS structures, as shown in Figure 5, and bandgap narrowing will increase charge in the substrate when a laser is used compared to the charge produced by heavy ions. A number of new submicron device structures have been proposed that use layers with high and low doping concentrations. For example, the lowimpurity transistor[16] shown in Figure 5 has highly doped wells underneath a low-impurity channel region. Much higher charge would be produced by a laser in the well region relative to that in the channel, compared to the charge produced by heavy ions. 


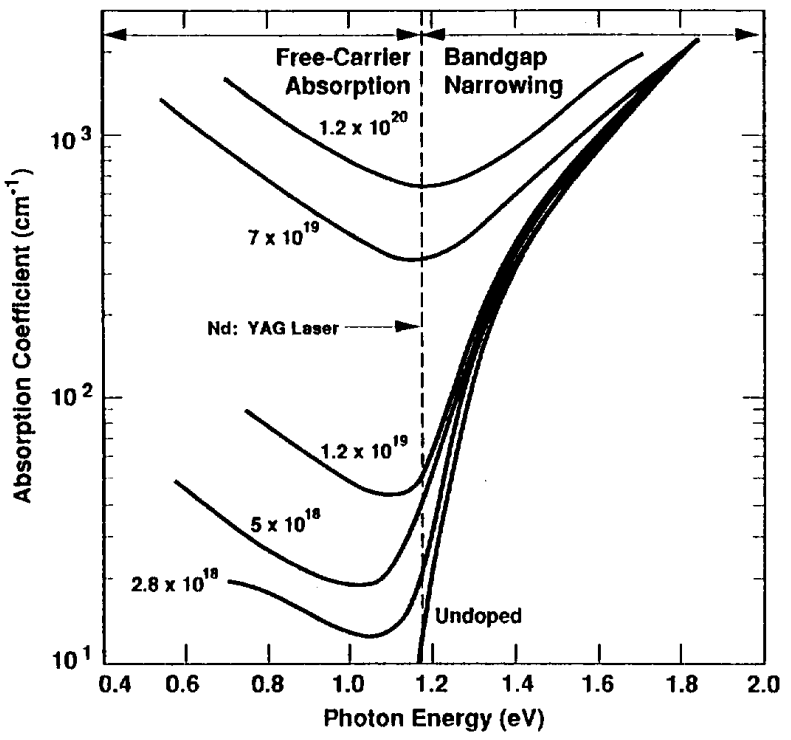

Figure 4. Bandgap Narrowing and Free-Carrier Absorption in Silicon (after Pantelides, Selloni, and Car, Reference 10).

\section{Carrier Density and Charge FunNeling}

Excess Carrier Density Profile. Although a laser can be used to create the same number of carriers in a thin region of a semiconductor as a heavy-ion track, the Rayleigh criterion limits the laser spot size to approximately $\lambda$. Thus, as shown in Figure 6, the peak excess carrier density of the laser near the surface of the semiconductor is approximately 200 times lower than that produced by a charged particle with a track diameter of $0.1 \mu \mathrm{m}$ (after thermalization). This not only affects

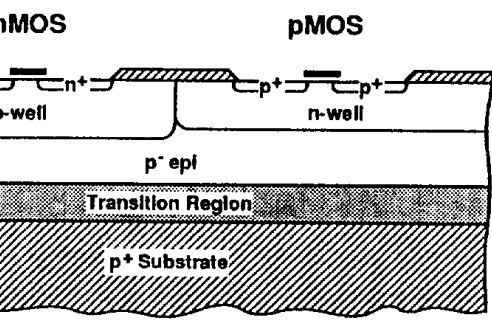

(a) Epitaxial CMOS Structure

Figure 5. Examples of CMOS Structures with Heavily Doped Regions Below the Surface Layer. funneling, but it also means that the laser carrier profile is equivalent to that of the charged particle after the much higher density of the charged particle has diffused so that the radius

of the plasma column is $\approx \lambda$. For lightly doped devices the difference in charge profile is usually unimportant, but it becomes increasingly significant for high carrier concentrations.

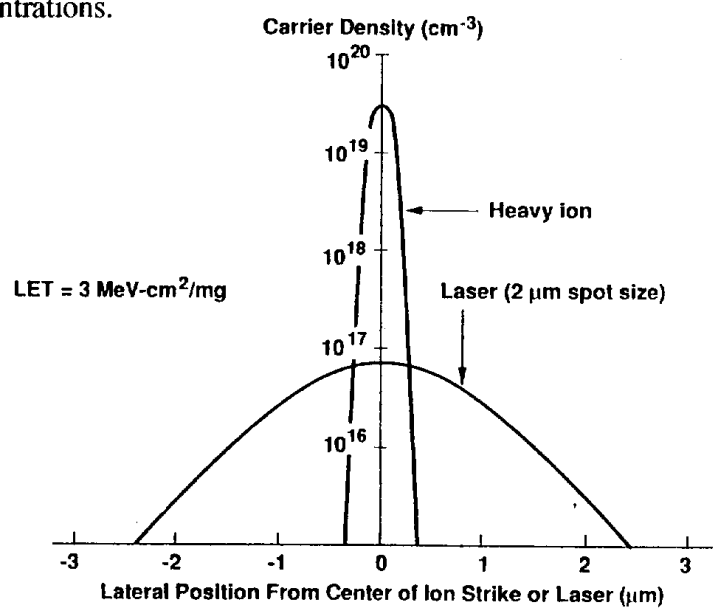

Figure 6. Comparison of Carrier Densities Generated by Lasers and Heavy Ions

The excess carrier profile is also affected by the optics that are used to produce a small beam. The usual technique is to use a microscope objective. A small focused spot can only be obtained by using an objective with high numerical aperture (N.A.). For a circular spot, the relationship between spot size, wavelength, and numerical aperture is

$d=0.61 \lambda(N . A$.$) ; the resolution of most lenses with$

nMOS

pMos

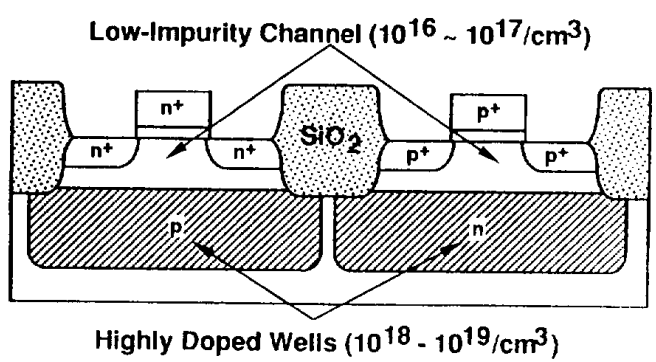

(b) Low-Impurity Channel Transistor for Advanced CMOS 
reasonable working distances does not achieve this value, and is limited to resolution of $\approx \lambda /$ (N.A.). With a high N.A., the light cone from the objective extends to large angles. Although refraction is reduced within the material because of the high refractive index of silicon, the angles are still large enough to cause the light to diverge rapidly with distance as it passes through the device. This places a further limitation on spot size; it causes the carrier density to be even lower in the deeper regions of the device that are important for funneling. Figure 7 shows the increase of the laser diameter for various distances in silicon as a function of numerical aperture. Note that the power density decreases as the square of the diameter, causing the effective LET to be lower by nearly a factor of 10 for a $6 \mu \mathrm{m}$ depth compared to the value at the surface for a high N.A. objective. This disagrees with the statement in Ref. 3 that the dimension of the laser track remains small to depths of $50 \mu \mathrm{m}$. However, they did not consider the entrance angle of the microscope objective in their analysis.

The laser beam can be focused to a small, localized region near the surface. However, the large entrance angle spreads the laser beam over extended regions in the substrate. Although the intensity is reduced, this charge can be collected by diffusion, and may cause much different responses for

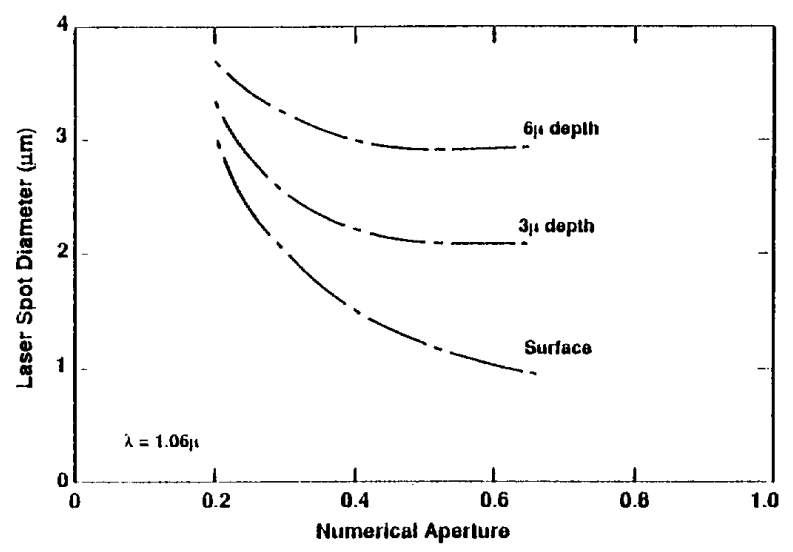

Figure 7. Increase in Diameter of Laser Track Due to Refraction in Silicon.

effects such as latchup and multiple-bit upset that are strongly affected by diffused charge compared to heavy-ion tests, which produce a much more localized carrier distribution in the substrate.

The finite laser spot size also becomes important as devices are scaled to smaller sizes. For devices with submicron feature sizes, a diffraction-limited laser spot is larger than critical device dimensions. This restricts the ability to use the laser as a microprobe.

Charge Funneling. Previous workers have noted that lasers with short pulse lengths (below $100 \mathrm{ps)} \mathrm{are} \mathrm{required} \mathrm{in}$ order to simulate charge funneling, $[2,3,17]$ which requires mode-locked lasers. Buchner et al.[4] evaluated funneling by measuring the intensity dependence of total charge, and concluded that funneling occurred with lasers because a $4 / 3$ power dependence on intensity was observed, as predicted for heavy ions. However, these experiments were limited to extremely high equivalent LET values $\left(50-400 \mathrm{MeV}-\mathrm{cm}^{2} / \mathrm{mg}\right)$ where the excess carrier density of the laser is well above the background concentration. They did not measure prompt charge (which is very difficult), but assumed that the nonlinearity of total charge was sufficient evidence of funneling.

Our calculations of charge collection with laser irradiation using the PISCES computer code show that because of the lower carrier density, funneling does not occur with low LET values. These calculations were made for a cylindrical junction, which eliminates the charge density problem that occurs for rectangular geometries when two-dimensional simulation programs are used. The track density of the laser was changed with distance to take refraction into account. Assuming that the depletion region does not collapse until the excess carrier density exceeds the background concentration, $[14,15]$ the funneling threshold depends on the doping level. Figure 8 shows the results of this work, taking into account the increased laser spot size at depths within the material. For lightly doped substrates, typical of older technologies with feature sizes of $2 \mu \mathrm{m}$ or larger, the funneling threshold is generally below the threshold LET for upset with heavy ions. Thus, for older devices, a pulsed laser will reasonably approximate heavy-ion effects in the region of interest. However, as feature sizes are reduced, substrate doping levels increase, and it becomes increasingly likely that the threshold LET for upset with heavy ions will be above the effective LET threshold for funneling with the laser. Once this threshold is crossed, calibration of the laser by correlation with heavy ion tests is no longer straightforward. For devices with doping levels above $10^{17} \mathrm{~cm}^{-3}$, the threshold LET must be above $10 \mathrm{MeV}-\mathrm{mg} / \mathrm{cm}^{2}$ in order for funneling to occur with a laser. This is an important result, as doping levels of this magnitude are required for devices with feature sizes below 1 $\mu \mathrm{m}$. If funneling does not occur, the prompt charge produced by the laser will be approximately a factor of three lower with the laser for p-type silicon substrates. 


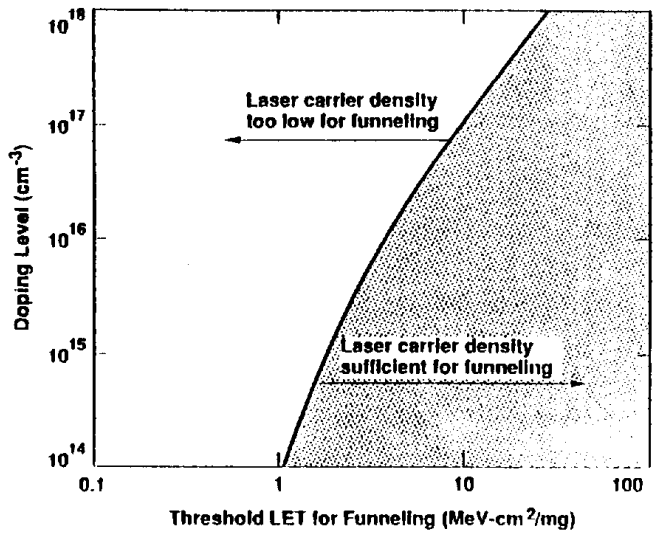

Figure 8. The Relationship Between Carrier Concentration and Funneling for Silicon.

\section{ADDITIONAL ABSORPTION PROCESSES}

At low intensities, the exponential absorption predicted by Beer's law clearly applies. However, extremely high power densities result when pulsed lasers are focused to small spots, and additional absorption mechanisms become significant at high power densities which cause nonlinearities in the light absorption. The most important mechanisms are free-carrier absorption and two-photon absorption.

Free-Carrier Absorption. Free-carrier absorption is the result of direct absorption of photons by carriers in the conduction or valence band. It does not produce ionization, but increases the energy of carriers within these bands. Thus, it is a loss mechanism that increases absorption in highly doped regions. It can significantly reduce the light intensity in regions below highly doped regions, such as contacts or source/drain junctions, and must be taken into account when using lasers in modern devices.

The free-carrier absorption coefficient increases with increasing wavelength.[20] For silicon at $1.06 \mu \mathrm{m}$, the absorption coefficient for this process becomes significant at about $2 \times 10^{18} \mathrm{~cm}^{-3}$. It increases proportionally with the carrier concentration, and also increases with wavelength. Its effect relative to band-to-band absorption was shown earlier in Figure 4. Absorption from this mechanism can be more than three orders of magnitude greater than fundamental band-toband absorption. The important point is that regions with high doping density will reduce the light intensity in regions beneath them because of absorption, and effectively shield infrared laser light from regions beneath them.

Two-Photon Absorption. The very high power density associated with focused lasers was noted earlier. An additional absorption mechanism involving simultaneous absorption of two photons can occur at high energy densities, and causes a highly nonlinear increase in the absorption.[21]
Two-phonon absorption (TPA) is so nonlinear that it has been used to fabricate optical limiters. TPA absorption can be described by the equation

$$
\begin{gathered}
\mathrm{I}_{0} \mathrm{e}^{-\alpha \mathrm{x}} \\
1+\left(\beta \mathrm{I}_{\delta} / \alpha\right)\left(1-\mathrm{e}^{-\alpha x}\right)
\end{gathered}
$$

where $\alpha$ is the usual absorption coefficient and $\beta$ is the TPA coefficient. For GaAs, $\beta$ is $23 \mathrm{~cm} / \mathrm{GW}$.[22] The TPA coefficient of silicon is expected to be between 30 and 40 . At low intensities, Equation 2 reduces to the usual exponential absorption expression. When the power density reaches $\approx 0.1$ $\mathrm{cm} / \mathrm{GHz}$, the absorption begins to increase sharply, and the effective value of $\alpha$ is much larger than that assumed from fundamental absorption, and effective LET no longer scales with optical power density.

The curves in Figure 9 show a calculation of the effective LET with laser irradiation (assuming initial calibration with the assumption of linear absorption, as in Eq. 1) with the LET from heavy-ion irradiation. The curves combine the funneling results from PISCES calculations and a calculation of twophoton absorption using Equation 2.. Funneling extensions of the depletion regions for $\mathrm{n}$ - and p-regions are taken from the literature.[18] The nonlinearities in effective LET at low LET values are due to the lower carrier density of the laser. The transition intensity for funneling depends on doping level and substrate type. Two-photon absorption becomes important at high LET values, increasing the absorption coefficient. This shows that the effective absorption of the laser is inherently nonlinear, compared to heavy ions, and that the nonlinearity overlaps the LET threshold region for many devices of current interest. For LET values lower than the LET transition for

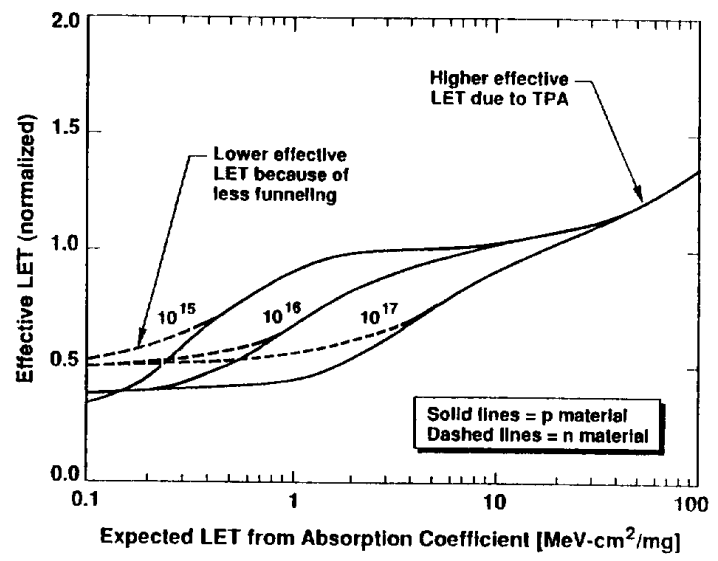

Figure 9. Dependence of Effective LET on Doping Concentration and Expected LET for a p-n Junction 
funneling, the effective LET is lower in a $p-n$ junction compared to the LET in the bulk substrate. Thus, the ratio between the diffusion and prompt charge will be substantially different with laser irradiation than with heavy ions, particularly for p-substrates.

\section{APPLICATIONS OF LASERS}

In many cases, lasers have been used to simulate singleevent effects without taking into account the differences between heavy ion tracks and pulsed lasers. Often the calibration is done by correlating laser and heavy-ion results, assuming that an SEU-like response from a circuit irradiated with a laser is equivalent to that of heavy ions. One frequent error is the use of wavelengths with inadequate penetration. For example, some workers have used a doubled Nd:YAG wavelength $(0.532 \mu \mathrm{m})$ in silicon devices; light at this wavelength is completely absorbed at the surface, and the resulting charge generation profile is quite different from that of a heavy-ion track. Another frequent error is to ignore the refraction of the laser beam in material. This is particularly important in devices with lightly doped substrates, where prompt charge collection depths can exceed $10 \mu \mathrm{m}$.

There are several key factors that need to be considered when using lasers for SEU simulation. First, the penetration depth must be long enough so that the light is absorbed uniformly over the charge collection depth. For silicon, this usually requires a wavelength between 0.95 and $1.06 \mu \mathrm{m}$. Second, although earlier work claimed that funneling occurs with the laser in the same way as heavy ions, this is only true at higher equivalent LET values. Third, the absorption coefficient will be different in highly doped regions of a device, with the result that the effective LET will be different in these regions than in lightly doped regions. It is important to know enough about the structure of devices that are being tested to determine which regions have high doping concentrations, and to take this into account when calibrating the laser and interpreting the results. Finally, there is the obvious fact that light from the laser cannot penetrate metallization regions. This is a serious drawback for technologies with double-level metallization.

Earlier work with wide-pulse lasers used irradiation from the back side to partially overcome the limitations of occlusion by metallization. This work involved global irradiation, flooding the entire integrated circuit with laser light.[23] At $1.06 \mu \mathrm{m}$, the penetration depth is sufficiently long to penetrate the entire wafer thickness. Furthermore, in this application it is not necessary to use a focused beam. Although this is attractive for global irradiation, SEU testing requires a dense, localized ionization track. Refraction of the laser beam in silicon would increase the effective spot size to $20-30 \mu \mathrm{m}$ after it passed through the wafer. Thus, this approach is not feasible for single-particle effects.
Although the previous sections of the paper have emphasized important differences between lasers and heavy ions, lasers have been successfully applied to a number of device types, primarily as diagnostic tools. The most frequent method used to calibrate lasers is to correlate the laser response of a device with tests from heavy ions. Most older technologies have doping concentrations in critical regions (such as the substrate and well for CMOS) that are below $10^{17}$ $\mathrm{cm}^{-3}$, which greatly diminishes the importance of the effects emphasized in this paper, and allows better quantitative agreement between lasers and heavy ions. However, it is worth noting that most investigators have relied on correlation tests alone, and have not attempted to relate their test results to the laser power.

One advantage of this correlation approach is that it eliminates the need to consider optical reflection losses. For the refractive index of these materials (3.4-3.5), approximately $30 \%$ of the incident light will be reflected at normal incidence, assuming an air/semiconductor interface. However, the surface passivation adds an additional dielectric layer, which may produce constructive or destructive interference, and makes the net surface reflection strongly dependent on passivation thickness. Furthermore, the focused laser beam is not at normal incidence. The net result is that optical transmission can vary from about 0.4 to 0.85 , depending on the interface structure.

Even in older structures, the carrier profile of the laser can be an important factor. Figure 10 compares the carrier profile of an Nd:YAG laser with that of a galactic cosmic ray particle as well as that of a heavy ion from an accelerator with a range of $40 \mu \mathrm{m}$. (This range is specified as a minimum by current test standards for single-particle effects). The approximate charge collection range for prompt charge (including funneling) and diffusion in lightly doped substrates is shown at the left of the Figure. There are two important points. First, the range of the particle from the accelerator is marginal for diffused charge, which means that the laser pulse may actually produce more charge for the same equivalent LET in processes such as latchup and multiple-bit upset that depend on diffused as well as prompt charge. If the accelerator ion is not at normal incidence, which is permitted by test standards, this difference is further enhanced. On the other hand, as noted in Figure 8 funneling is markedly reduced for moderate LET values and high doping levels, and in this case a heavy ion would produce significantly more charge than the laser pulse. Thus, lasers may produce more or less charge than an "equivalent" heavy ion, depending on the doping level and the importance of diffused charge. The second point is that the laser pulse spreads laterally because of refraction. Thus, the laser pulse is not very localized, which may limit the ability to probe small regions. This is particularly important for newer devices which may have feature sizes of about half the diameter of the focused laser. 
Uncertainties in surface reflection and track structure, along with occlusion by surface metal layers, limit absolute comparisons of lasers and heavy ions to about $50 \%$ for older devices, and differences of about this amount have frequently been observed in the literature.

For newer devices, the much higher doping levels increase the importance of track structure and nonlinear absorption in different layers. This will add additional uncertainty to laser simulation, particularly if devices with different doping profiles are compared. However, for controlled situations, such as hardness assurance testing which involves comparisons of laser-induced SEU response on devices fabricated with the same process, the differences in quantitative agreement between heavy ions and the laser are less important.

In using lasers for hardness assurance, it is important to keep in mind that most single-event phenomena are strongly affected by the precise location of the laser strike. In order to make comparisons between different devices, it is generally necessary to mount devices on a two-axis movable platform. The mechanical resolution of the fixture must be less than 1 $\mu \mathrm{m}$ in order to locate the beam precisely on the test device. A hardness assurance procedure must include a well documented method of defining the precise region of a device in which the laser is to be located. A detector that is sensitive in the near infrared region can be used to locate the position of the laser. Considerable effort is required in order to set up the mechanical and optical hardware for hardness assurance tests, but they are far more convenient than particle accelerators, particularly if large numbers of parts are to be compared over long time periods.

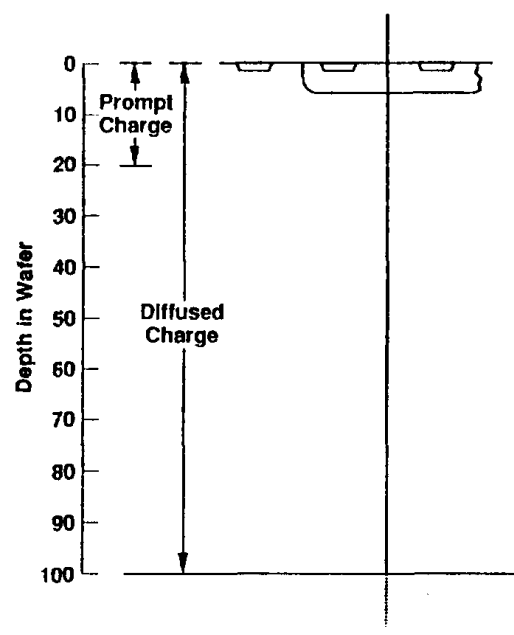

(a) Galactic Cosmlc Ray

ic

\section{(b)}

Figure 10. Comparison of Charge Deposition Profiles of Galactic Cosmic Rays, Heavy Ions from a Test Facility, and the
Lasers are also very useful as diagnostic tools. The advantages of time synchronization, microprobing, convenience and cost mentioned in the Introduction are not directly affected by the correlation and calibration factors discussed in earlier sections. However, it is vital that users of lasers understand the basic structure of the devices that they are testing, along with the inherent limitations of laser simulation.

\section{SUMMARY AND CONCLUSIONS}

This paper has compared fundamental charge generation processes for lasers and heavy ions. Several factors need to be taken into account when using lasers that become more important as devices are scaled to smaller dimensions, and were not considered in earlier laser studies. At high doping concentrations bandgap narrowing causes the absorption coefficient to vary in different regions of a device, with the result that the same light intensity will produce a different LET in different regions. This is particularly important in devices with extended regions of high carrier density, such as epitaxial substrates. It is also very important in GaAs because of the much steeper slope of the absorption coefficient and more limited penetration depth of tunable dye lasers, which have lower power densities.

Fundamental optical considerations limit the diameter of a focused laser spot to about $1.5 \mu \mathrm{m}$. The resulting carrier density is about 200 times lower than that of a heavy-ion track. This reduces funneling at high carrier concentrations, even when a laser with very short pulse length is used. This is extremely important, as it affects calibration and correlation of laser effects. It also contradicts earlier claims that funneling Pulse from a $1.06 \mu \mathrm{m}$ Focused Laser. 
was essentially the same for lasers and heavy ions. Decreased funneling is particularly significant for scaled devices with threshold LET values below $10 \mathrm{MeV}-\mathrm{mg} / \mathrm{cm}^{2}$, because of the high doping levels. At high laser power densities, additional mechanisms that cause nonlinear absorption become important, further complicating interpretation of laser results.

Although the laser is still a very useful probing tool, the factors discussed above limit the ability to establish quantitative comparisons between lasers and heavy ions. They also place restrictions on the types of phenomena that can be studied with lasers. It is important to realize the differences between lasers and heavy ions in order to use lasers as effective diagnostic tools.

\section{References}

1. D. H. Habing, "The Use of Lasers to Simulate RadiationInduced Transients in Semiconductor Devices and Circuits," IEEE Trans. Nucl. Sci., NS-12, No. 5, 91 (1965).

2. S. Buchner, et al., "Laser Simulation of Single-Event Upsets," IEEE Trans. Nucl. Sci, NS-34, 1228 (1987).

3. A. K. Richter and I. Arimura, "Simulation of Heavy Charged Particle Tracks Using Focused Laser Beams," IEEE Trans. Nucl. Sci, NS-34, 1234 (1987).

4. S. Buchner, et al., "Charge Collection from Focused Laser Pulses," IEEE Trans. Nucl. Sci, NS-35, 1517 (1988).

5. S. Buchner, et al., "Pulsed Laser Induced SEU in Integrated Circuits: A Practical Method for Hardness Assurance Testing," IEEE Trans. Nucl. Sci, NS-37, 1825 (1990).

6. W. C. Dash and R. Newman, "Intrinsic Optical Absorption in Single-Crystal Germanium and Silicon at $77 \mathrm{~K}$ and $300 \mathrm{k}$," Phys. Rev., 99, 1151 (1955)

7. H. R. Phillip and E. A. Taft, "Optical Constants of Silicon in the Region 1 to $10 \mathrm{eV}, "$ Phys. Rev. Lett., $\underline{8}, 13$ (1962).

8. J. I. Pankove, Optical Processes in Semiconductors, PrenticeHall, New Jersey, 1971.

9. D. E. Hill, "Infrared Transmission and Fluroescence of Doped Gallium Arsenide," Phys. Rev., 133, A866 (1964).

10. B. W. Hughlock, et al., "Ion-Induced Charge Collection in GaAs MESFETs and Its Effect on SEU Vulnerability," IEEE Trans. Nucl. Sci, NS-38, 1442 (1991).

11. W. Kolasinski, R. Koga, E. Schnauss and J. Duffey, "The Effect of Elevated Temperature on Latchup and Bit Errors in CMOS Devices," IEEE Trans. Nucl. Sci., NS-33, 1605 (1986).

12. J. L. Pankove, Optical Processes in Semiconductors, PrenticeHall, New Jersey, 1971.

13. H. Casey, Jr., D. D. Sell, and K. W. Wecht, "Concentration Dependence of the Absorption Coefficient for $n$ - and $\mathrm{p}$-Type GaAs between 1.3 and $1.6 \mathrm{eV}$, , J. Appl. Phys., 46, 250 (1975).

14. S. Pantelides, A. Selloni and R. Car, "Energy Gap Reduction in Heavily Doped Silicon: Causes, and Consequences," Solid State Elect., 28, 17 (1985).

15. J. R. Brews, et al., "Generalized Guide for MOSFET Miniaturization," IEEE Elect. Dev. Lett., EDL-1, 2 (1980).
16. M. Nagata, "Limitations, Innovations, and Challenges of Circuits and Devices into a Half Micrometer and Beyond," IEEE J. Solid St. Circuits, 27, 465 (1002)

17. C. A. Gossett, B. W. Hughlock, and A. H. Johnston, "Laser Simulation of Single-Event Effects," IEEE Trans. Nucl. Sci., NS-39, 1647 (1992).

18. T. R. Oldham, F. B. McLean, and J. M. Hartman, "Revised Funnel Calculations for Heavy Particles with High dE/dx," IEEE Trans. Nucl. Sci, NS-33, 1646 (1986).

19. L. D. Edmonds, "A Simple Estimate of Funneling-Assisted Charge Collection," IEEE Trans. Nucl. Sci, NS-38, 828 (1991).

20. J. P. Woerdman, "Some Optical and Electrical Properties of a Laser-Generated Free-Carrier Plasma in Si," Philips Res. Rept. Suppl. 7 (1971).

21. T. Boggess, et al., "Nonlinear-Optical Energy Regulation by Nonlinear Refraction and Absorption in Silicon," Optics Lett., 2, 291 (1984).

22. E. W. Van Stryland, et al., "Two-Photon Absorption, Nonlinear Refraction, and Optical Limiting in Semiconductors," Opt. Eng., 24, 613 (1985).

23. E. E. King, et al., "Transient Radiation Screening of Silicon Devices Using Backside Laser Irradiation," IEEE Trans. Nucl. Sci, NS-29, 1809 (1982). 